\title{
Paper-supported thin-layer ion transfer voltammetry for ion detection
}

\author{
Jiawang Ding ${ }^{\mathrm{a}, \mathrm{b}}$, Thomas Cherubini ${ }^{\mathrm{b}}$, Dajing Yuan ${ }^{\mathrm{b}}$, Eric Bakker ${ }^{\mathrm{a}, *}$ \\ ${ }^{a}$ Yantai Institute of Coastal Zone Research, Chinese Academy of Sciences, Yantai, Shandong 264003, P. R. China \\ b Department of Inorganic and Analytical Chemistry, University of Geneva, Quai Ernest-Ansermet 30, CH-1211 Geneva, Switzerland
}

A R T I C L E I N F O

\section{Keywords:}

Ion transfer voltammetry

Thin-layer

Paper-supported

Ion detection

\begin{abstract}
A B S T R A C T
We report here on paper-supported thin sample layer voltammetry for the determination of ions. To achieve this goal, a simple setup for the coupling of a commercially available electrode to a silver rod electrode was designed and evaluated for paper-supported thin-layer voltammetry. Linear scan ion transfer voltammetry was explored here for ion-selective membranes doped with an ionophore. The ion-transfer processes and electrochemical behaviors of the system are here evaluated and confirmed by numerical simulation. In the proof-of-concept experiments described, the ions tetrabutylammonium chloride $\left(\mathrm{TBA}^{+}\right)$and potassium $\left(\mathrm{K}^{+}\right)$were studied as model analytes at membranes without and with ionophore, respectively. A linear relationship from $0.1 \mathrm{mM}$ to $1.0 \mathrm{mM} \mathrm{K}^{+}$was obtained between the charge and ion concentration. The coexistence of background sodium ions did not give appreciable interference, but the background wave was not completely isolated from the analyte wave, as also confirmed by the model. The methodology was successfully demonstrated for determination of $\mathrm{K}^{+}$ in mineral water. It is anticipated that this paper-supported thin-layer detection approach may provide an attractive readout protocol for disposable paper-based analytical devices as the methodology does not place strict demands on reference electrode performance.
\end{abstract}

\section{Introduction}

With rapid progress in the field of paper-based analytical devices, potentiometric ion sensing and biosensing platforms have also been explored by making use of paper-based microfluidic sampling and functionalized paper substrates [1-4]. While these versatile potentiometric devices may offer adequate sensitivity and selectivity, they utilize open circuit potential (OCP) as a readout for a single measurement. This requires excellent potential stability, as the Nernst equation already dictates errors of $4 \%$ and $8 \%$ for just a $1 \mathrm{mV}$ potential uncertainty in the assessment of monovalent and divalent ions, respectively. For $3 \mathrm{mV}$, the error increases to over $12 \%$ and $26 \%$, respectively, which is already unacceptable for most analytical applications, as in routine clinical diagnostics. An important reason for this is the difficulty to incorporate a reliable reference electrode into a paper-based format, although recent progress with ionic liquid based reference electrode concepts has been encouraging [5,6]. It would therefore be desired to explore alternative transduction principles in a paper based format where the reference electrode is less important on sensor output.

Exhaustive coulometry is, ideally, an absolute measurement principle based on the strict proportionality between the concentration of the analyte and the involved charge. For cases of excellent selectivity and conversion efficiency, meaning that only the analyte, but all of it, is consumed, the approach should, in agreement with Faraday's law, allow for a calibration-free measurement.

By employing a thin-layer sample for the coulometry, the complete electrolysis can be achieved reasonably rapidly, in the time scale of seconds to minutes. Since the introduction of thin-layer coulometry for determination of redox-active species [7], its applications have expanded to non-electroactive ionic species based on ion transfer at the aqueous/organic solution interface [8-12]. In order to improve its selectivity, thin-layer coulometry based on ion-selective membrane is currently being pursued by our group and others [13-16].

In recent years, studies in the area of coulometry have focused on the use of thin-layer ionophore-based membranes [17-19]. Meanwhile, important progress has occurred in the design of suitable thin-layer cells with the introduction of new materials $[12,13,16,20]$. Our group designed a custom-made microfluidic electrochemical cell based on tubular materials, in which a thin layer sample is confined between an inner $\mathrm{Ag} / \mathrm{AgCl}$ wire and the outer microporous hydrophobic tube doped with the ion-selective component [16]. The electrochemical response behavior of the thin-layer coulometric sensors under a controlled potential is elucidated and the membrane response model was developed by using a numerical simulation [21]. Besides the incorporation of an

\footnotetext{
* Corresponding author.

E-mail address: eric.bakker@unige.ch (E. Bakker).
} 
inert physical separator for cell response improvement [22], a double pulse technique was utilized for background correction [23]. By using the proposed controlled-potential thin-layer coulometry, a number of different ions including potassium, calcium, protamine, as well as nitrate, and nitrite have been measured [24].

Using the above mentioned thin-layer configuration, coulometric sensors for halides were also designed in our group by using Nafion as a permselective cation-exchanger membrane $[25,26]$. In contrast to the controlled potential coulometry, the application of cyclic voltammetry or a linear sweep potential to thin layer samples was explored for multianalyte halide detection. As evidenced by these studies, the proposed methodology is absolute and potentially calibration-free. Clearly, cyclic voltammetry promises to expand the scope of coulometry based on thin-layer samples, but its applicability for coulometry based on ionselective membranes doped with an ionophore has not yet been investigated. Moreover, sensing the target ions in this manner with a simplified experimental setup may be challenging but worthwhile.

Paper is attractive for the stated purpose, as it may transport the sample to the detection compartment by its wicking properties, and additionally create an aqueous layer of well-defined thickness that is suitable for thin-layer coulometry [27]. Indeed, our previous research indicated that a paper-based thin-layer coulometric sensor based on the formation of silver halide precipitates can be used for halide determination in real samples [26]. Unlike the conventional potentiometric measurements based on the linear dependence between the logarithm of the ion activity and the OCP, the obtained charge is directly proportional to analyte concentration for coulometric measurements, which makes coulometry more suitable for detecting small analyte concentration changes. If the approach is efficient and chemically selective, the resulting signal should be temperature-insensitive, absolute and potentially calibration-free [28]. These are important potential advantages with batch-fabricated disposable single-use analytical devices such as paper-based analytical devices.

Herein, we report on a paper-supported thin-layer ion transfer voltammetry for the determination of ions. To realize this concept, we developed a system in which a commercial electrode body mounted with an ion-selective membrane doped into microporous polypropylene (Celgard) and a silver rod were used as working and reference/counter electrode, respectively. Thin-layer ion transfer voltammetry was evaluated on tetrabutylammonium chloride $\left(\mathrm{TBA}^{+}\right)$and potassium $\left(\mathrm{K}^{+}\right)$as representative analytes.

\section{Experimental section}

\subsection{Materials}

Sodium tetrakis[3,5-bis-(trifluoromethyl)phenyl]borate (NaTFPB), Potassium tetrakis(4-chlorophenyl)borate (KTpClPB), potassium ionophore I (Valinomycin), dodecyl 2-nitrophenyl ether (DDNPE), tetrabutylammonium chloride $\left(\mathrm{TBA}^{+}\right.$), potassium chloride, tetrahydrofuran (THF), Whatman filter papers 2 (90-mm diameter, 8 - $\mu \mathrm{m}$ pore size, 190 $\mu \mathrm{m}$ thickness), and Whatman filter papers 3 (qualitative) were purchased from Sigma-Aldrich. Porous polypropylene membranes (Celgard membrane $20 \mu \mathrm{m}$ thickness) were kindly provided by Membrana, Wuppertal, Germany and used as a supporting material. The cocktail for $\mathrm{TBA}^{+}$impregnation of the Celgard membranes contained $90 \mathrm{mg}$ of DDNPE (a more lipophilic plasticizer compared to the $o$-NPOE to avoid leaching) and $10 \mathrm{mg}$ of NaTFPB. The membrane cocktail for $\mathrm{K}^{+}$was composed of $97.8 \mathrm{mg}$ of DDNPE, $1.8 \mathrm{mg}(16 \mathrm{mmol} / \mathrm{kg})$ of valinomycin, and $0.4 \mathrm{mg}$ of KTpClPB ( $25 \mathrm{~mol} \%$ relative to the ionophore). Aqueous solutions were prepared by dissolving the appropriate salts in Milli-Qpurified distilled water.

\subsection{Experimental setup}

Cyclic voltammograms were obtained on a PGSTAT $302 \mathrm{~N}$ potentiostat (Metrohm Autolab, Utrecht, The Netherlands) controlled by a personal computer using Nova 1.8 software (supplied by Autolab). A piece of silver (with a diameter of $5.0 \mathrm{~mm}$ and a thickness of ca $2.0 \mathrm{~mm}$ ) was attached to a stainless steel rod by using conductive glue. The home-made silver rod was electrochemically coated with $\mathrm{AgCl}$ and used as a reference/counter electrode. Electrode bodies (OSTEC, Oesch Sensor Technology, Sargans, Switzerland) were used to mount the Celgard membranes. All experiments were carried out at room temperature.

For $\mathrm{TBA}^{+}$determination, the inner solution was $10^{-3} \mathrm{M} \mathrm{TBA}^{+}$with $10^{-2} \mathrm{M} \mathrm{NaCl}$. All experiments were performed in $10 \mathrm{mM} \mathrm{NaCl}$ background, unless otherwise indicated. For $\mathrm{K}^{+}$measurements, $10^{-3} \mathrm{M} \mathrm{KCl}$ with $10^{-3} \mathrm{M} \mathrm{NaCl}$ was used as inner filling solution. Prior to the experiments, the membrane was conditioned in the same solution that was also used as the inner filling solution of the electrode. The membranes were then mounted onto electrode bodies as they are used commonly for ion-selective electrodes.

\subsection{Preparation of the detection cell}

In previous research, we designed a simple paper-based electrochemical cell for halide determination [24]. Experiments revealed that the stability and performance of the cell is not adequately suited for coulometric ion sensing using ionophore-based membranes: the free hanging ion-selective membrane is not sufficiently rigid to define a constant thin layer sample volume. In the present work, a simplified setup that has compatibility with a commercial electrode body was designed, which can also improve the stability of the ion sensor. As shown in Scheme $1 \mathrm{~A}$ to $\mathrm{C}$, the custom-built setup consists of three parts: (1) $\mathrm{Ag}$ rod coated with $\mathrm{AgCl}$ (Scheme $1 \mathrm{~A}-\mathrm{e}$ ), as a reference/ counter electrode, allowing the oxidation/reduction modulated transfer of ions in the sample, (2) a commercial electrode body (Scheme 1 A-d) with cocktail impregnated Celgard membrane (Scheme $1 \mathrm{~A}-\mathrm{a}$ ), allowing transfer of target ions across the membrane to the inner solution with high selectivity, and (3) filter paper, which is used for thin-layer sampling. For the working electrode, a home-made porous Teflon pad (Scheme $1 \mathrm{~A}-\mathrm{c}$ ) was placed on the bottom of the Celgard membrane, which places the membrane in close contact with the sample solution and the Ag rod. In addition, to avoid the mechanical failure of the ionselective membrane, we introduce a paper layer (Scheme $1 \mathrm{~A}-\mathrm{b}$, a diameter of $7.0 \mathrm{~mm}$, Whatman filter papers 3 ) between the membrane and the porous support.

If not otherwise specified, a piece of Whatman filter paper 2 (a diameter of $5.0 \mathrm{~mm}$ ) was placed on the silver rod. $3.0 \mu \mathrm{L}$ sample solution was added on the Whatman filter paper 2 for measurements. For practical applications, a $6.0 \mathrm{~mm}$ wide gap was designed on the top of the commercial electrode cap to insert the filter paper for sampling via capillary wicking (as shown in Scheme 1B). A single filter paper $(0.6 \mathrm{~cm} \times 4.0 \mathrm{~cm})$ was used for sampling. A $5 \mathrm{~min}$ exposure time ensured that the sample liquid wicked through the paper. The filter paper was dipped into a small beaker containing the sample solution during the entire analysis. A new piece of paper was used for each standard and sample solution.

\subsection{Real sample analysis}

The potassium concentration in mineral water (obtained from local supermarket) was determined using the standard addition method. Specifically, $100 \mu \mathrm{L}$ of mineral water was added into $900 \mu \mathrm{L}$ background solution with $1.0 \mathrm{mM} \mathrm{NaCl}$ and different amounts of added $\mathrm{KCl}$ $(0,0.25,0.50$, and $0.75 \mathrm{mM}) .3 .0 \mu \mathrm{L}$ sample solution was added on a filter paper (Whatman filter paper 2 with a diameter of $5.0 \mathrm{~mm}$ ) for measurements. A new piece of paper was used for each sample solution. The concentration of potassium was also measured by atomic emission spectroscopy. Potassium was measured at a wavelength of $766.5 \mathrm{~nm}$ with a slit width of $0.2 \mathrm{~nm}$. 

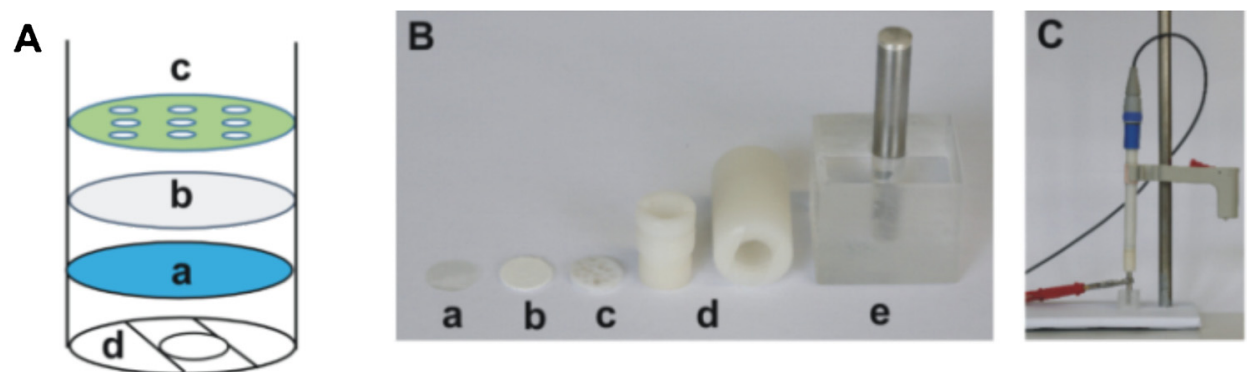

Scheme 1. (A) Scheme of the electrochemical set up used in this work. (B) and (C) are the photographs of the setup. (D) Illustration of the electrochemical mechanism of the sensing processes. With this configuration, a $3 \mu \mathrm{L}$ sample solution was dropped onto the filter paper sample holder for analysis, thereby precisely defining the amount of sample.
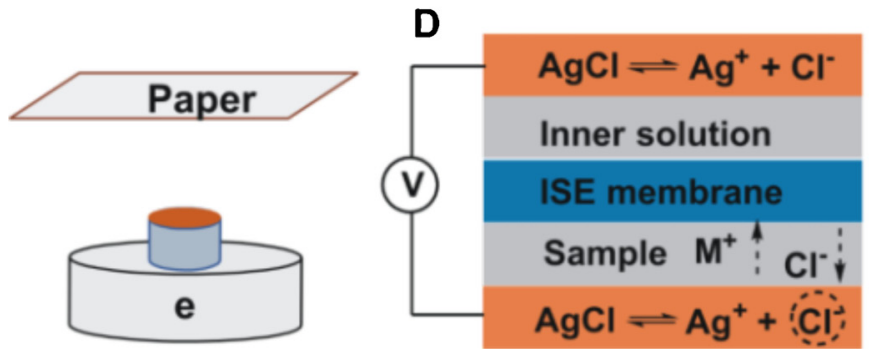

\subsection{Theoretical model}

The treatment follows a recent procedure based on one dimensional diffusion kinetics that improves on the earlier approach given by Morf $[29,30]$. As in previous work, a one dimensional space $(x)$ is subdivided into finite distance elements of spacing $\Delta \mathrm{x}$ that evolve in a time (t) grid with time distance $\Delta \mathrm{t}$. Elements $-\mathrm{x}_{\mathrm{aq}}$ to 0 and 1 to $\mathrm{x}_{\mathrm{mem}}$ represent the elements for the aqueous layer and membrane, respectively.

By treating the sample-membrane interface using the phase boundary potential equation, the difference between the applied potential $\left(E_{\text {app }}\right)$ and the open circuit potential (OCP) can be obtained without consideration of accumulation and depletion in the inner solution, see also reference [22]:

$E_{A P P}-O C P=\operatorname{slog} \frac{C_{[0, t]}}{C_{[0,0]}} \frac{C_{[1,0]}}{C_{[1, t]}} \frac{C_{\left[x_{m}, t\right]}}{C_{\left[x_{m}, 0\right]}}$

where $s$ is the Nernstian slope and $c_{[p o s i t i o n, 0]}$ are the initial concentrations. The potential scan ( $\nu$ is the scan rate) is performed in a defined interval of time $\left(0 \leq \mathrm{t} \leq \mathrm{t}_{\max }\right)$, which modulates the applied potential starting from the initial potential $\left(\mathrm{E}_{\text {init }}\right)$ as follows:

$E_{\text {app }}-O C P=E_{\text {init }}-v t, 0 \leq t \leq t_{\max }$

Taking an interfering ion $J$ of the same charge as the primary ion into consideration, the following ion-exchange equilibrium can be formulated [18]:

$\frac{c_{[1, t]}}{c_{[1, t]}+c_{J[1, t]}}=\frac{c_{[0, t]}}{c_{[0, t]}+K_{I J}^{\text {pot }} c_{J, 0}}$

Where $K_{I J}^{p o t}, c_{J, 0}$ are the selectivity coefficient and the concentration (strictly, activity) of the interfering ion in the aqueous phase. Since the sum of $c_{1, t}$ and $c_{J, 1, t}$ dictates the ion-exchanger concentration of the membrane $\left(R_{m}\right)$, the last membrane element is given as follows:

$c_{x_{m e m, t}}=2 R_{m}-\frac{c_{[0, t]}+K_{I J}^{\text {pot }} c_{[J, 0]}}{c_{[0, t]}} c_{[1, t]}$

The relationship is inserted into Eq. 1 to give:

$c[1, t]=\frac{2 R_{m}}{1+\left(K_{I J}^{\text {pot }} c_{[I, 0]}+C_{\text {initial }} 10^{\left(E_{\text {init }}-v t\right.}\right) / c[0, t]}$

The parameters used here were a sample-layer thickness of $200 \mu \mathrm{m}$, a membrane thickness of $50 \mu \mathrm{m}$, diffusion coefficients in the sample, $D_{\mathrm{a}}$, and membrane, $D_{\mathrm{m}}$, of $10^{-5} \mathrm{~cm}^{2} \mathrm{~s}^{-1}$ and $10^{-7} \mathrm{~cm}^{2} \mathrm{~s}^{-1}$, respectively and an ion-exchanger concentration, $R_{m}$, of $5.0 \mathrm{mM}$; finite distance steps, $d$, of $10 \mu \mathrm{m}$; time steps, $\Delta \mathrm{t}$, of $1 \mathrm{~ms}$ and scan rate of $5 \mathrm{mV} \mathrm{s}^{-1}$. All

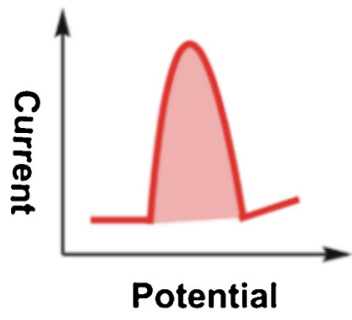

calculations were performed in Mathematica 10 (Wolfram Research).

\section{Results and discussion}

The aim of this work is to develop a potentially general, simple and reliable paper-supported thin-layer ion sensor with ion-selective membrane. To this end, a simplified experimental setup was developed and applied for ion sensing (Scheme 1A-C). The experimental setup has two main advantages: First, it enables measurements using the commercially available electrode with ion-selective membrane. Second, thinlayer solutions can be adjusted by using different filter papers. The principle of the proposed ion sensor is shown in Scheme 1D. Cyclic voltammetry, which is a valuable technique for the study of electrochemical mechanisms was used to study the transfer of the target ions across the sensing membrane [12]. When an anodic potential scan is applied at the electrode, the background chloride ions confined in the paper are electrodeposited on the silver electrode, while the cation will selectively transfer from the confined sample across the ion-selective membrane to the inner solution of the electrode. The ion transfer process between inner solution and ISE membrane is opposite to that of the sample solution and ISE membrane. If quantitative and exhaustive transfer of the target ions can be achieved within the potential window, the paper-supported thin-layer ion transfer voltammetry could be used for calibration-free analysis.

In our initial experiments, the tetrabutylammonium cation, an ion of relatively high lipophilicity and a common model species for liquid/ liquid ion transfer was selected to achieve an adequate experimental setup. Porous Celgard membrane doped with lipophilic plasticizer and cation-exchanger was prepared. $10^{-3} \mathrm{M} \mathrm{TBA}^{+}$with $10^{-2} \mathrm{M} \mathrm{NaCl}$ was used as inner solution, which keeps the potential at the $\mathrm{Ag} / \mathrm{AgCl}$-inner solution and membrane-inner solution from changing significantly during measurements. These relatively high concentrations should remain unperturbed during the measurement.

The $\mathrm{TBA}^{+}$extraction into and out of the membrane layer triggered by the oxidation-reduction of $\mathrm{Ag} / \mathrm{AgCl}$ was first studied in bulk solution. As shown in Fig. 1, with increasing applied potential, the oxidation of $\mathrm{Ag}$ results in the cation transfer from the sample to the inner solution of the electrode. Increasing the hydrophilicity of the ions results in an increasing potential to transfer ions across the membrane [31]. Therefore, the cation exchanger membrane with its higher selectivity in the Hofmeister series exhibits a high preference for $\mathrm{TBA}^{+}$ over other ions in the sample. Indeed, the transfer of $\mathrm{TBA}^{+}$is obtained at a lower potential in comparison with $\mathrm{Na}^{+}$(Fig. 1). Based on the above, $\mathrm{TBA}^{+}$is established as the main contributor to ion-transfer 


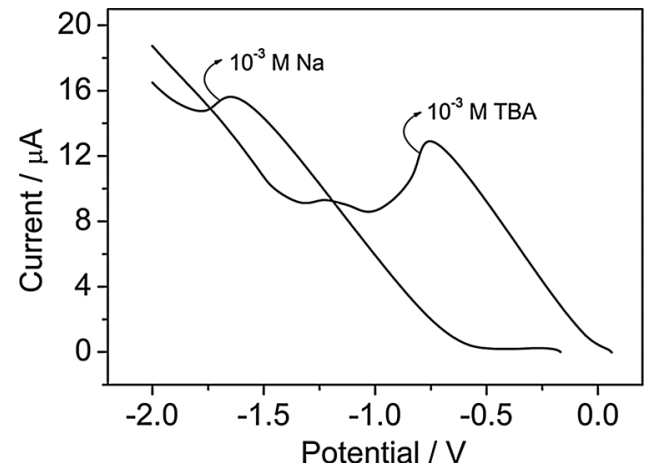

Fig. 1. Cyclic voltammograms obtained in bulk solutions. The inner solution was $10^{-3} \mathrm{M}$ tetrabutylammonium chloride with $10^{-2} \mathrm{M} \mathrm{NaCl}$. The sample solutions contain the target ions without supporting electrolyte.

behavior of the sample and may be measured selectively in the presence of $\mathrm{Na}^{+}$by cyclic voltammetry. This current may in principle force the anions to move in the opposite direction, but the cation-exchanger in the membrane suppresses the extraction of anions from the inner solution into the membrane. The potential was consequently backscanned to the initial potential to reverse the process and guarantee the reproducible responses for the oxidation of $\mathrm{Ag}$ and the unperturbed state of the membrane.

For exhaustive thin-layer cyclic voltammetry, all target ions should be consumed/transferred within the potential window. The scan rate may therefore play an important role for the exhaustive measurement. Fig. 2A presents cyclic voltammograms for $2.5 \times 10^{-4} \mathrm{M} \mathrm{TBA}^{+}$at different scan rates $\left(1-10 \mathrm{mV} \mathrm{s}^{-1}\right)$. The peak current is linear with scan rate, which is consistent with an exhaustive extraction, also called thin layer behavior (Fig. 2B). It should be noted that the separation between forward and reverse peaks is $320 \mathrm{mV}$, which is affected by both the aqueous-solid and aqueous-membrane interfaces (Fig. S1). At first glance, this separation would indicate an irreversible process but may instead be given by relatively long diffusion times in the two phases
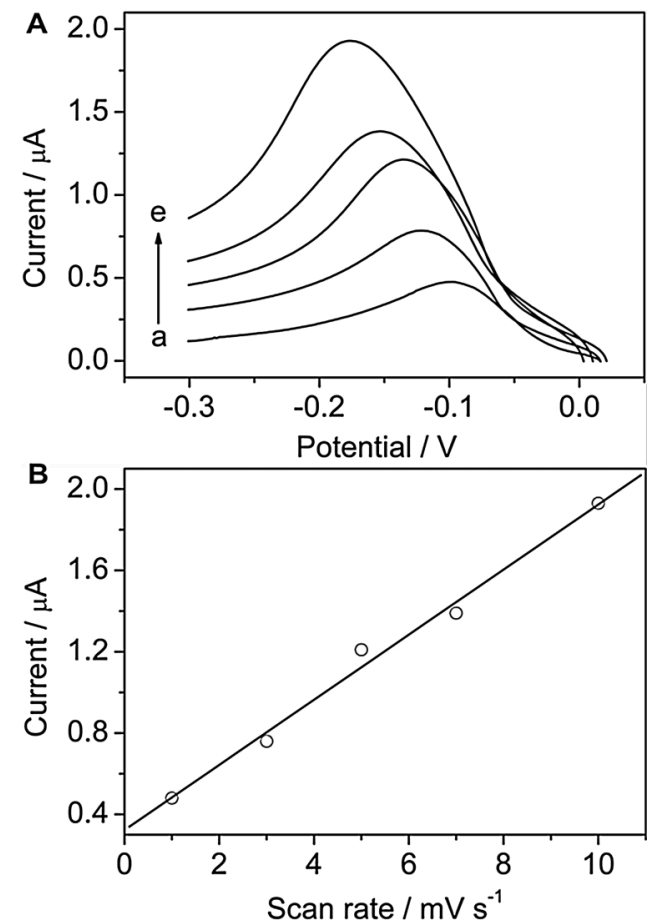

Fig. 2. (A) Cyclic voltammograms obtained for $2.5 \times 10^{-4} \mathrm{M}$ TBA and $10^{-2} \mathrm{M}$ $\mathrm{NaCl}$ with different scan rate $\left(1,3,5,7,10 \mathrm{mV} \mathrm{s}^{-1}\right.$ from a to e). (B) Peak current as a function of scan rate.
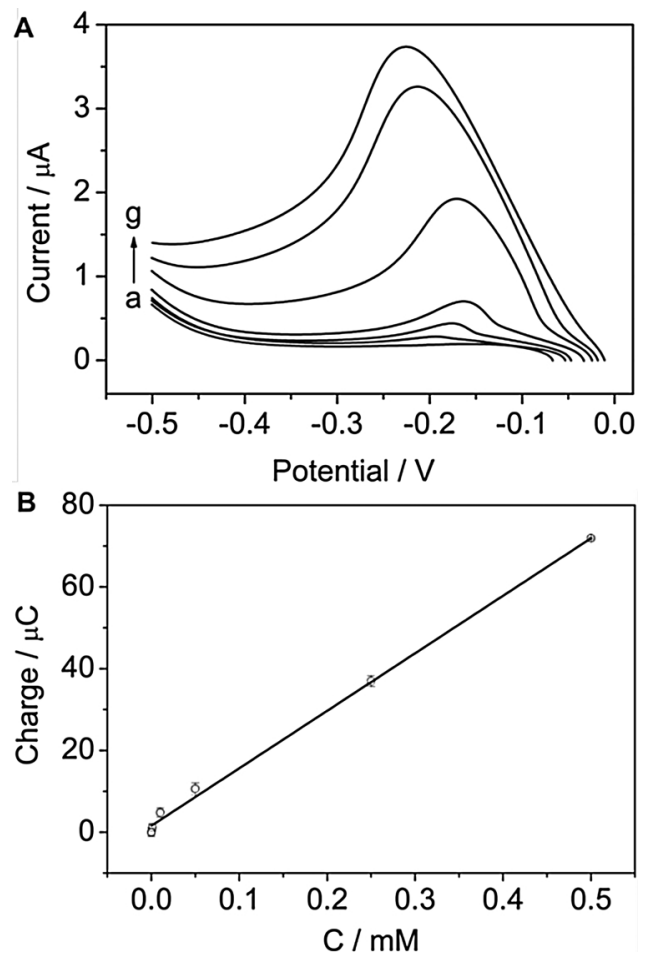

Fig. 3. (A) Cyclic voltammograms obtained for different concentrations of TBA with a scan rate of $10 \mathrm{mV} \mathrm{s}^{-1}$. (a), $0.01 \mathrm{M} \mathrm{NaCl}$, and $0.01 \mathrm{M} \mathrm{NaCl}$ with different concentrations of TBA, $1.0 \times 10^{-6}$ (b), $1.0 \times 10^{-5}$ (c), $5.0 \times 10^{-5}$ (d), $2.5 \times 10^{-4}$ (e), $5.0 \times 10^{-4}(\mathrm{f})$, and $1.0 \times 10^{-3}(\mathrm{~g}) \mathrm{M}$ TBA. (B) Calibration curve for TBA with a scan rate of $10 \mathrm{mV} \mathrm{s}^{-1}$. Error bars represent one standard deviation for three measurements.

(aqueous sample and organic membrane). The effect of sample volume on the electrochemical responses was also studied in the range from 1.5 to $5.0 \mu \mathrm{L}$. As expected, the observed charge increased with larger sample volume up to $5.0 \mu \mathrm{L}$, which indicates the effective transfer of the $\mathrm{TBA}^{+}$across the membrane. For a $5.0 \mathrm{~mm}(190-\mu \mathrm{m}$-thickness) filter paper, the sampling volume was $3.7 \mu \mathrm{L}$ without considering the porosity of the paper. To achieve a wider concentration range, $3.0 \mu \mathrm{L}$ of sample solution was used in all subsequent experiments.

Fig. 3A shows the cyclic voltammograms obtained with the setup to which $3.0 \mu \mathrm{L}$ sample solution containing different concentrations of $\mathrm{TBA}^{+}$was added. The overall charge at various concentration differences was determined from current integration of the linear scans in Fig. 3A. If the $\mathrm{TBA}^{+}$ions are exhaustively transferred, the remaining $\mathrm{TBA}^{+}$concentration in the thin-layer paper approaches zero. In that case, a linear relationship is expected between the charge and total $\mathrm{TBA}^{+}$concentration, which is indeed observed (Fig. 3B). Other cations such as $\mathrm{Na}^{+}, \mathrm{K}^{+}, \mathrm{H}^{+}$, and $\mathrm{Ca}^{2+}$ did not exhibit any significant increase of current (data are not shown). Similar results were also obtained using paper sampling via capillary action (Fig. S2). It should be noted that exhaustive extraction can be achieved in concentrations lower than $10^{-5} \mathrm{M}$. However, tailing of peak current can be observed at negative potential at a high sample concentration (Fig. 3A), which indicates that exhaustive transfer of all amount of ion is not realized. Indeed, Faraday's law dictates that the total electric charge passed through the electrode should be $14.5 \mathrm{mC}$ for $0.05 \mathrm{mM} \mathrm{TBA}^{+}$and a $3.0 \mu \mathrm{L}$ sample volume, while the integrated charge at $10 \mathrm{mV} \mathrm{s}^{-1}$ was found as $12.1 \mathrm{mC}$. This gives a $84 \%$ coulometric efficiency. When the $\mathrm{TBA}^{+}$concentration reaches $0.5 \mathrm{mM}$, only a $50 \%$ coulometric efficiency can be obtained.

A deviation from thin-layer behavior may result in a deviation from linearity at a high sample concentration. Moreover, the effective sample volume is smaller than the added one, which may be due to the electrostatic adsorption of $\mathrm{TBA}^{+}$on paper. Despite this, the setup is simple 

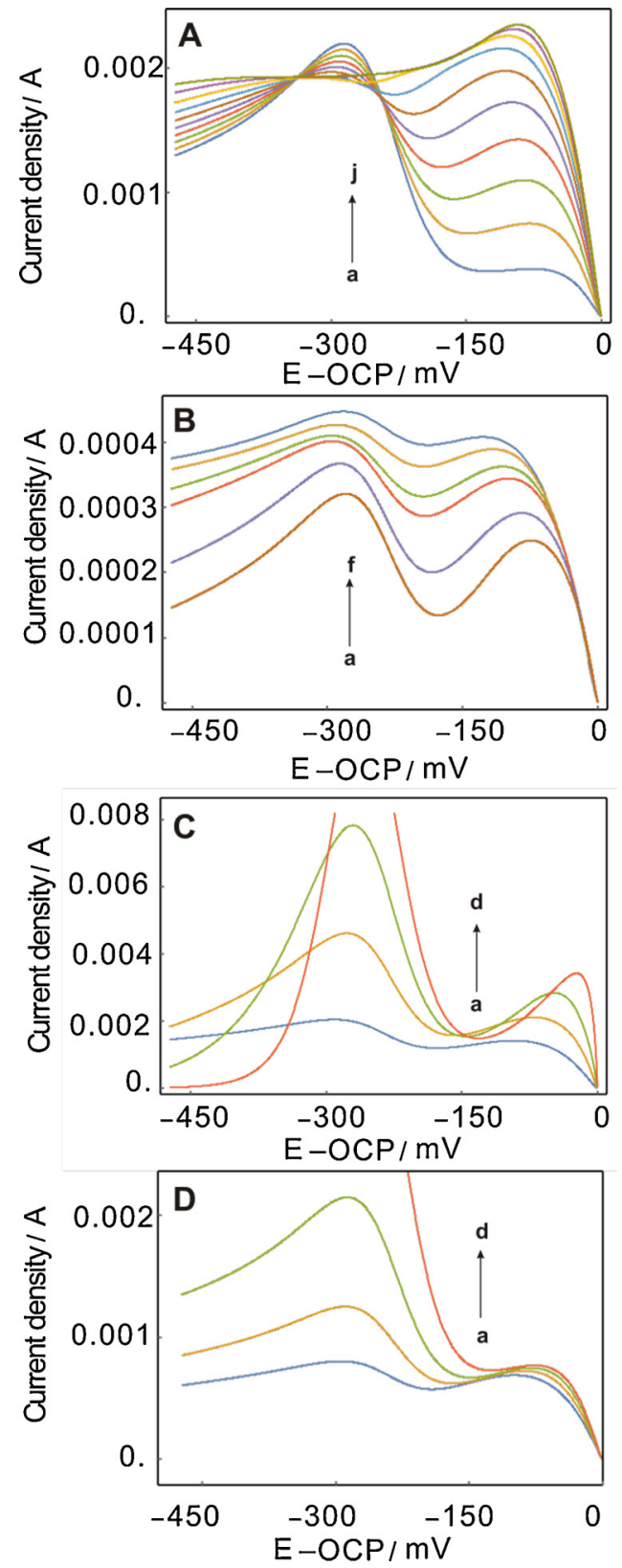

Fig. 4. Calculated cyclic voltammograms obtained for (A) different concentrations of $\mathrm{KCl}(0.1 \mathrm{mM}$ (a) to $1.0 \mathrm{mM}(\mathrm{j})$, the concentration increment was $0.1 \mathrm{mM}$ ); (B) different scan rate ( $\nu=2$ (a), 3 (b), 5 (c), 6 (d), 8 (e), and $10 \mathrm{mV}$ $\mathrm{s}^{-1}(\mathrm{f})$ ); (C) different diffusion coefficients $\left(D_{\mathrm{m}}=10^{-7}(\mathrm{a}), 5 \times 10^{-7}(\mathrm{~b}), 10^{-6}(\mathrm{c})\right.$, and $10^{-5} \mathrm{~cm}^{2} \mathrm{~s}^{-1}(\mathrm{~d})$ ), and (D) different concentrations of ion-exchanger (2 (a), 3 (b), 5 (c), and $10 \mathrm{mM}$ (d)). Parameters: sample-layer thickness, $\delta_{\mathrm{a}}=200 \mu \mathrm{m}$; membrane thickness, $\delta_{\mathrm{m}}=50 \mu \mathrm{m}$; diffusion coefficients in the sample and membrane, $D_{\mathrm{a}}=10^{-5} \mathrm{~cm}^{2} \mathrm{~s}^{-1}$ and $D_{\mathrm{m}}=10^{-7} \mathrm{~cm}^{2} \mathrm{~s}^{-1}$, respectively; ion-exchanger concentration, $R_{\mathrm{m}}=5.0 \mathrm{mM}$; finite distance steps, $d=10 \mu \mathrm{m}$; time steps, $\Delta t=1 \mathrm{~ms}$. Scan rate: $5 \mathrm{mV} \mathrm{s}^{-1}$. Sample analyte concentration, $\mathrm{c}(0,0)=$ $200 \mu \mathrm{M}$.

and effective for paper-based thin-layer voltammetry. In addition, background corrections can be used to eliminate undesired interference, which will be discussed below. While $\mathrm{TBA}^{+}$was selected as a model here, other comparatively hydrophobic ions such as perchlorate or ionic drugs may be detected using the paper-supported system.

The facilitated ion transfer of hydrophilic ions with ionophorecontaining membranes was subsequently investigated. To our knowledge, thin-layer ion sensors with ionophore-based membranes have scarcely been investigated using cyclic voltammetry. Herein, a potassium-selective system based on valinomycin was selected as a model. In order to evaluate the thin-layer voltammetry for the determination of ions, the characteristics of the sensing system are modeled by numerical simulation. For simplicity, all sample-independent potential contributions were neglected in the course of the sensing protocol.

The theoretical voltammograms for different concentrations of potassium ions were simulated without considering the ohmic drop. As shown in Fig. 4A, the current of the first (potassium dependent) peak increases linearly with the concentration of potassium. At higher scan potentials, the ion transfer can no longer be assisted by the ionophore, and background ions start to extract into the membrane. Therefore, an obvious peak appearing at ca $-300 \mathrm{mV}$ is ascribed to the transfer of sodium ions. At higher concentrations of $\mathrm{K}^{+}$, the sodium peak decreases gradually and eventually almost disappears in the potential window owing to the larger quantity of the $\mathrm{K}^{+}$that needs to be transferred. Even so, the background wave was not completely isolated from the analyte wave. Therefore, the target ion-transfer process was not exhaustive with $100 \%$ coulometric efficiency. The theoretical prediction was consistent with the experimental results cited below. The resolution between the two peaks could be further improved with decreasing scan rates. Plots of the voltammograms curves with different scan rates were calculated (Fig. 4B). As expected, peak resolution becomes more pronounced as the scan rate is decreased. However, complete isolation of the two waves was not observed even at a scan rate of $2 \mathrm{mV} \mathrm{s}^{-1}$.

The simulated cyclic voltammograms with different membrane diffusion coefficients were also demonstrated. As predicted theoretically (Fig. 4C), the current increases significantly with the increase of the membrane diffusion coefficient. Moreover, the position of the peaks shifts to more positive potential and an enhancement of peak resolution can be obtained. Therefore, this method places important demands on the design of the membrane materials, which have an impact on the electrochemical behaviors of the system. Other porous supporting materials resulting in larger diffusion coefficients may be alternative options. Moreover, increasing the amount of the lipophilic ion-exchanger $\left(R_{m}\right)$ also resulted in an increase of peak current (Fig. 4D). With the simulation tool, the performance of the sensors can be predicted and improved for the proposed methodology.

In this study, the ion transfer process places great demands on the selectivity of the membrane. As shown in the simulated cyclic voltammograms, the coexistence of background sodium ions did not give appreciable interference, but the background wave was not completely isolated from the potassium wave. Therefore, the contributions from the undesired interference may be reduced via background correction. Two different approaches for background subtraction were proposed. For the first approach ("inflection point" approach), the inflection point for the background wave can be found. The slope at the point was extrapolated down to zero current to calculate the charge. The obtained charge was then used for the background correction (Fig. S3 A). The charge is linear up to the point where the background wave is similar in height to the sample wave. At higher concentrations, an obvious deviation from the linear range can be observed (Fig. S3B). As a simple comparative approach ("cut off" approach), by integrating the charge up to the minimum between the sample wave and background wave in the voltammogram, the total charge can be obtained and used for background compensation (Fig. 5A). Compared to the first protocol, a good linear relationship between the charge and the concentration can be observed even at higher concentrations for the simple "cut off" approach (Fig. 5B). Therefore, the "cut off" approach may be used to partially reduce the undesired interference.

The concept was then evaluated experimentally with a potassiumselective membrane. The ion transfer voltammetry was again first explored in bulk solution. As shown in Fig. 6, an obvious (ca. $300 \mathrm{mV}$ ) potential difference was observed for the transfer of target ion $\left(\mathrm{K}^{+}\right)$and background ion $\left(\mathrm{Na}^{+}\right)$. As in the presence of the ionophore, the transfer of $\mathrm{K}^{+}$is obtained at a lower potential in comparison with $\mathrm{Na}^{+}$owing to 

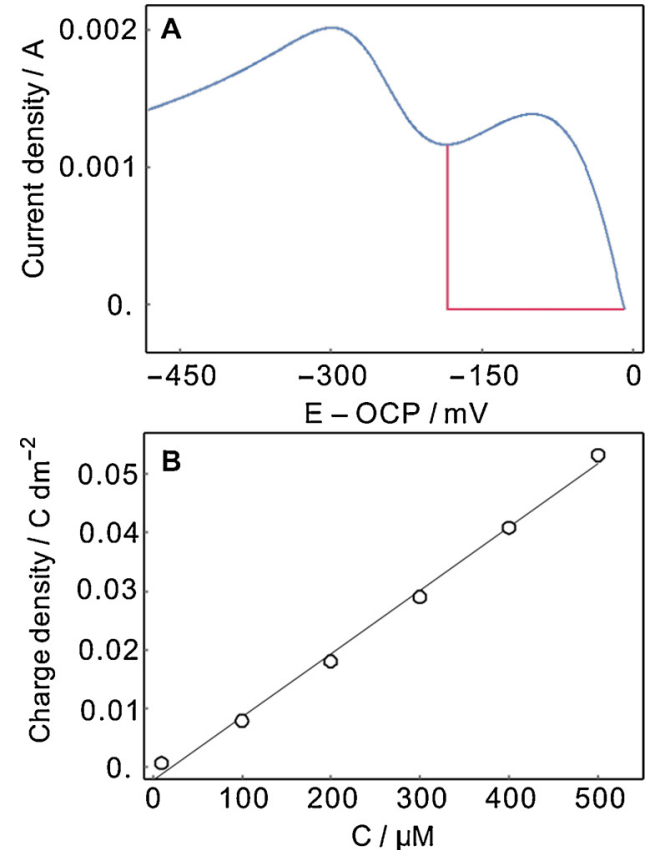

Fig. 5. (A) Background subtraction method based on the "cut off" approach. The straight red line indicates the area for charge calculations. The Calculated cyclic voltammogram was obtained for $0.4 \mathrm{mM} \mathrm{KCl}$ with $1.0 \mathrm{mM} \mathrm{NaCl}$. All other parameters as for Fig. 4. (B) Background-corrected charge obtained from the simulated results by using the "cut off" approach. (For interpretation of the references to colour in this figure legend, the reader is referred to the web version of this article).

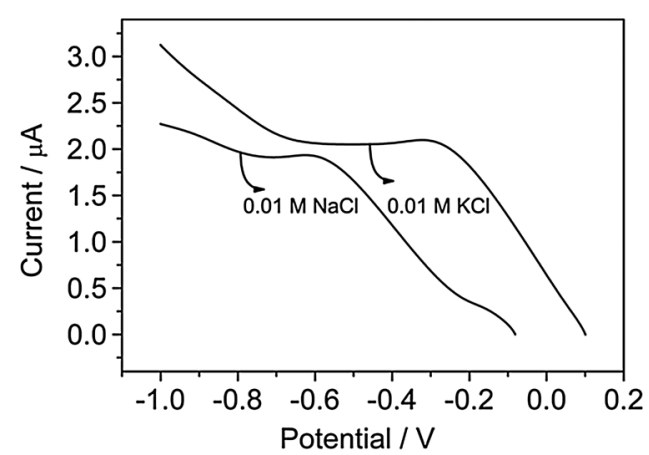

Fig. 6. Cyclic voltammograms obtained in bulk solutions. The inner solution was $10^{-3} \mathrm{M} \mathrm{KCl}$ with $10^{-3} \mathrm{M} \mathrm{NaCl}$. The sample solutions contain the target ions without supporting electrolyte.

the lower free energy of transfer and the higher binding affinity between ionophore and analyte. The separation of the two observed transfer peaks, which is dependent on the selectivity of the membrane, allows the proposed methodology for potassium detection.

The electrochemical response behavior of the sensor with a papersupported thin-layer solution was investigated. For the thin-layer sample, ion-transfer of the target ions may occur in a potential window that depends on membrane selectivity. During the forward scan, the $\mathrm{K}^{+}$ undergoes assisted ion transfer into and across the ion-selective membrane by the ionophore. At higher potentials, solution background ions are also extracted and deteriorate the selectivity of the membrane. Indeed, two peaks are clearly distinguished. The peak at $-350 \mathrm{mV}$ is ascribed to the transfer of $\mathrm{K}^{+}$while the one at $-650 \mathrm{mV}$ to that of $\mathrm{Na}^{+}$. While sodium ions did not give appreciable interference, the analyte wave was not completely isolated from the background wave. This phenomenon was consistent with the theoretical results mentioned above (Fig. 4A). The close match between the calculated data and the
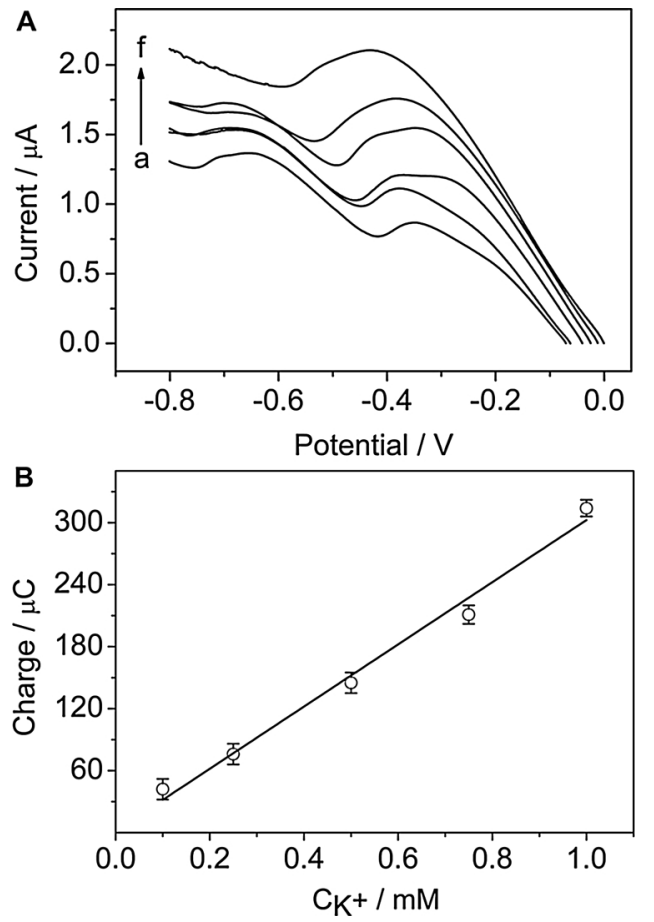

Fig. 7. (A) Cyclic voltammograms obtained for different concentrations of $\mathrm{KCl}$ with a scan rate of $2 \mathrm{mV} \mathrm{s}^{-1}$. (a, $0.001 \mathrm{M} \mathrm{NaCl}$, and $0.001 \mathrm{M} \mathrm{NaCl}$ with different concentrations of $\mathrm{KCl}, 1.0 \times 10^{-4}$ (b), $2.5 \times 10^{-4}$ (c), $5.0 \times 10^{-4}$ (d), $7.5 \times 10^{-4}$ (e), and $1.0 \times 10^{-3} \mathrm{M}$ (f) $\mathrm{KCl}$. (B) Calibration curve for $\mathrm{K}^{+}$with a scan rate of $2.0 \mathrm{mV} \mathrm{s}^{-1}$. Error bars represent the standard deviation for three measurements.

experimental data shows that the model adequately predicts the thin sample layer voltammetry.

Despite the similarities in response behavior between experimental and calculated voltammograms, the actual peaks are broader because of the contribution of the other processes to the electrochemical reaction. As predicted by the simulation (Fig. 4B), peak resolution can be improved with decreasing the scan rate. Indeed, with a slow scan rate $\left(2 \mathrm{mV} \mathrm{s}^{-1}\right)$, the sensor was found to adequately respond to potassium (Fig. 7A), and a linear relationship between integrated charge (as indicated below) and the concentration of added potassium was found from $100 \mu \mathrm{M}$ to $1.0 \mathrm{mM}$ (Fig. 7B). It should be noted that the tailing of peak current at negative potentials indicates that the complete transfer of all ion is not realized. However, as shown in the simulated results, background corrections can be used to reduce undesired interference. Even though both of the two approaches give a value for the charge that is lower than the theoretically expected charge, experimental measurements reveal that better results can be obtained for the simpler approach (Fig. S4). The values for the charge are almost consistent with the theoretically expected charge when the potassium concentrations were lower than $0.75 \mathrm{mM}$. Therefore, the simple "cut off" approach may be a good choice for background correction and used for quantitative analysis.

It is interesting to note that the observed open circuit potentials of the membrane correspond to a Nernstian response $(61.6 \pm 4.0 \mathrm{mV})$ and can be converted to the potassium (Fig. S5). Compared to controlled potential coulometry, cyclic voltammetry can provide more useful information about the membrane process. For the situation in which exhaustive transfer is not achieved, the reporting of current peak height may be a good alternative choice. Using this experimental configuration, any presence of a spontaneous leakage of potassium should negatively affect the detection limit of the sensor (as indicated the presence of the current peak at $-0.35 \mathrm{~V}$ for the background voltammogram). The potassium leakage may also arise from the tight contact 

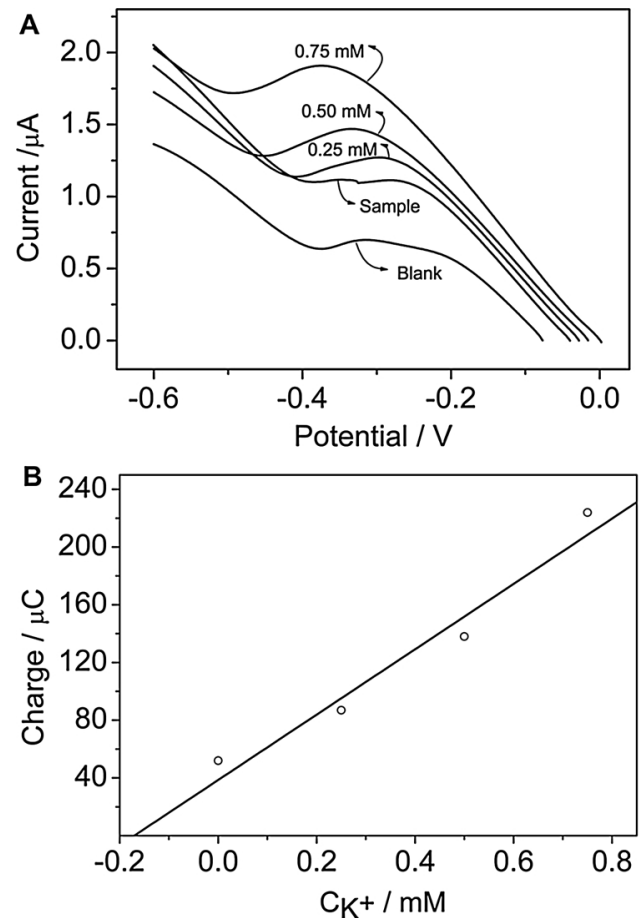

Fig. 8. (A) Cyclic voltammograms obtained for $\mathrm{K}^{+}$in mineral water with standard addition method. (B) Calibration line used to determine the $\mathrm{K}^{+}$concentration.

between the silver rod and the membrane. It is therefore expected that the linear dynamic range and the low detection limit could be further improved by optimizing the sensor design. For practical applications, the dynamic range may be adjusted by using different filter papers and different concentrations of sample solution. The reproducibility was found to be less than 5\% RSD, which may originate in the variations of the thickness and sampled concentrations for each new filter paper.

As an early stage application, the proposed method was used to measure the potassium concentration in mineral water, with Fig. 8A showing the corresponding cyclic voltammograms. The background subtracted charge was used for quantification. The potassium concentration in the mineral water was determined as $66.5 \pm 1.9 \mathrm{mg} / \mathrm{L}$ by standard addition calibration (as shown in Fig. 8B). The result was in accordance with the value obtained by atomic emission spectroscopy $(63.8 \pm 1.0 \mathrm{mg} / \mathrm{L})$ and the labelled value $(66.0 \mathrm{mg} / \mathrm{L})$, which confirms the viability of the proposed method.

\section{Conclusions}

In short, we have demonstrated the concept of paper-supported thin-layer voltammetry for ion sensing. The setup chosen here combines paper sampling and a commercial electrode based on ion-selective membrane for thin-layer voltammetry. Unlike a conventional constant potential coulometric measurement, cyclic voltammetry is applied to modulate the ion transfer across an ionophore based ion- selective membrane. The proposed sensor can provide not only consumed charge but also gives useful information about the membrane such as open circuit potential and selectivity. Of particular interest is that the iontransfer voltammetry with $100 \%$ electrolysis efficiency may be integrated with paper-based thin-layer sampling for potentially calibration-free analysis. Moreover, a simple and reasonable "cut off" approach was used for quantitative analysis in order to minimize the contribution of the background electrolyte to the total charge. While this study focused only on potassium detection based on paper sampling, this technique may allow for potentially calibration-free detection of other target ions and find valuable applications in batch- fabricated disposable single-use analytical devices. With the introduction of alternative materials and an adapted design, the performance of this type of sensors may be further improved.

\section{Acknowledgments}

The authors thank the Swiss National Science Foundation (NSF) for supporting this research. Jiawang Ding gratefully acknowledges the support by the China Scholarship Council and the National Natural Science Foundation of China (21575158).

\section{Appendix A. Supplementary data}

Supplementary material related to this article can be found, in the online version, at doi:https://doi.org/10.1016/j.snb.2018.10.046.

\section{References}

[1] W.J. Lan, X.U. Zou, M.M. Hamedi, J.B. Hu, C. Parolo, E.J. Maxwell, P. Bühlmann, G.M. Whitesides, Paper-based potentiometric ion sensing, Anal. Chem. 86 (2014) 9548-9553.

[2] J.W. Cui, G. Lisak, S. Strzalkowska, J. Bobacka, Potentiometric sensing utilizing paper-based microfluidic sampling, Analyst 139 (2014) 2133-2136.

[3] J.B. Hu, A. Stein, P. Bühlmann, A disposable planar paper-based potentiometric ionsensing platform, Angew. Chem. Int. Ed. 55 (2016) 7544-7547.

[4] J.W. Ding, B.W. Li, L.X. Chen, W. Qin, A three-dimensional origami paper-based device for potentiometric biosensing, Angew. Chem. Int. Ed. 55 (2016) 13033-13037.

[5] T. Kakiuchi, T. Yoshimatsu, N. Nishi, New class of $\mathrm{Ag} / \mathrm{AgCl}$ electrodes based on hydrophobic ionic liquid saturated with AgCl, Anal. Chem. 79 (2007) 7187-7191.

[6] T.T. Zhang, C.Z. Lai, M.A. Fierke, A. Stein, P. Bühlmann, Advantages and limitations of reference electrodes with an ionic liquid junction and three-dimensionally ordered macroporous carbon as solid contact, Anal. Chem. 84 (2012) 7771-7778.

[7] A.J. Bard, Electroanalysis and coulometric analysis, Anal. Chem. 40 (1968) 64-70.

[8] C. Sánchez-Pedreno, J.A. Ortuno, J. Hernández, Chronocoulometric flow-injection analysis with solvent polymeric membrane ion sensors, Anal. Chim. Acta 459 (2002) 11-17.

[9] S. Sawada, M. Taguma, T. Kimoto, H. Hotta, T. Osakai, Complete electrolysis using a microflow cell with an oil/water interface, Anal. Chem. 74 (2002) 1177-1181.

[10] Y. Yoshida, S. Yamaguchi, K. Maeda, Conducting polymer coated electrode as a counter and reference electrode in an organic phase and its application to a twoelectrode type thin-layer cell for voltammetry at liquid/liquid interface, Anal. Sci. 26 (2010) 137-139.

[11] G. Herzog, Recent developments in electrochemistry at the interface between two immiscible electrolyte solutions for ion sensing, Analyst 140 (2015) 3888-3896.

[12] Y. Yoshida, S. Nakamura, J. Uchida, A. Hemmib, K. Maeda, A flow electrolysis cell with a thin aqueous phase and a thin organic phase for the absolute determination of trace ionic species, J. Electroanal. Chem. 707 (2013) 95-101.

[13] A. Yoshizumi, A. Uehara, M. Kasuno, Y. Kitatsuhi, Z. Yoshida, S. Kihara, Rapid and coulometric electrolysis for ion transfer at the aqueous/organic solution interface, J. Electroanal. Chem. 581 (2005) 275-283.

[14] V. Bhakthavatsalam, E. Bakker, Selective coulometric release of ions from ion selective polymeric membranes for calibration-free titrations, Electroanalysis 20 (2008) 225-232.

[15] S. Amemiya, Y. Kim, R. Ishimatsu, B. Kabagambe, Electrochemical heparin sensing at liquid/liquid interfaces and polymeric membranes, Anal. Bioanal. Chem. 399 (2011) 571-579.

[16] E. Grygolowicz-Pawlak, E. Bakker, Thin layer coulometry with ionophore based ion-selective membranes, Anal. Chem. 82 (2010) 4537-4542.

[17] M. Kasuno, Y. Kakitani, Y. Shibafuji, T. Okugaki, K. Maeda, T. Matsushita, S. Kihara, Rapid and precise coulometric determination of calcium based on electrolysis for ion transfer at the aqueous|organic solution interface, Electroanalysis 21 (2009) 2022-2029.

[18] M.G. Afshar, G.A. Crespo, E. Bakker, Coulometric calcium pump for thin layer sample titrations, Anal. Chem. 87 (2015) 10125-10130.

[19] S. Jansod, M.G. Afshar, G.A. Crespo, E. Bakker, Alkalinization of thin layer samples with a selective proton sink membrane electrode for detecting carbonate by carbonate-selective electrodes, Anal. Chem. 88 (2016) 3444-3448.

[20] D. Dorokhin, G.A. Crespo, M.G. Afshar, E. Bakker, Low-cost thin layer coulometric microfluidic device based on ion-selective membrane for calcium determination, Analyst 139 (2014) 48-51.

[21] E. Bakker, Membrane response model for ion-selective electrodes operated by controlled potential thin-layer coulometry, Anal. Chem. 83 (2011) 486-493.

[22] A. Shvarev, B. Neel, E. Bakker, Detection limits of thin layer coulometry with ionophore based ion-selective membranes, Anal. Chem. 84 (2012) 8038-8044.

[23] E. Grygolowicz-Pawlak, A. Numnuam, P. Thavarungkul, P. Kanatharana, E. Bakker, Interference compensation for thin layer coulometric ion-selective membrane electrodes by the double pulse technique, Anal. Chem. 84 (2012) 1327-1335.

[24] M. Cuartero, G.A. Crespo, E. Bakker, Thin layer samples controlled by dynamic electrochemistry, CHIMIA 69 (2015) 203-206. 
[25] M. Cuartero, G.A. Crespo, M.G. Afshar, E. Bakker, Exhaustive thin-layer cyclic voltammetry for absolute multianalyte halide detection, Anal. Chem. 86 (2014) 11387-11395.

[26] M. Cuartero, G.A. Crespo, E. Bakker, Paper-based thin-layer coulometric sensor for halide determination, Anal. Chem. 87 (2015) 1981-1990.

[27] Y.Y. Yang, E. Noviana, M.P. Nguyen, B.J. Geiss, D.S. Dandy, C.S. Henry, Paperbased microfluidic devices: emerging themes and applications, Anal. Chem. 89 (2017) 71-91.

[28] E. Bakker, Can calibration-free sensors be realized? ACS Sens, 1 (2016) 838-841.

[29] D.J. Yuan, E. Bakker, Overcoming pitfalls in boundary elements calculations with computer simulations of ion selective membrane electrodes, Anal. Chem. 89 (2017) 7828-7831.

[30] W.E. Morf, E. Pretsch, N.F. De Rooij, Computer simulation of ion-selective membrane electrodes and related systems by finite-difference procedures, J. Electroanal. Chem. 602 (2007) 43-54.

[31] M. Velický, K.Y. Tam, R.A.W. Dryfe, Mechanism of ion transfer in supported liquid membrane systems: electrochemical control over membrane distribution, Anal. Chem. 86 (2014) 435-442.

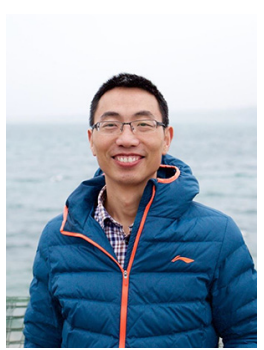

Jiawang Ding received his doctor's degree from Yantai Institute of Coastal Zone Research, Chinese Academy of Sciences in 2011 . He is currently working as an associate professor at the same institute. From 2016 to 2017, he did his joint researches at Åbo Akademi University (Finland) and Geneva University (Switzerland). His research interests include electrochemical sensors and biosensors.

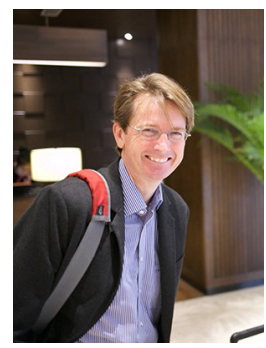

Eric Bakker obtained his doctoral degree at ETH Zurich and spent 14 years in the United States and 3 years in Australia before returning to Switzerland in 2010 as chair of Analytical Chemistry at the University of Geneva. His research is in chemical sensors, using electrochemistry and optics to achieve a sensitive and robust detection of electrically charged species. In recent years the field of application has been environmental systems. His papers have been globally cited about 20,000 times. Among his awards is the 2014 RSC Robert Boyle Prize in Analytical Science. He has published more than 300 papers and is an Associate Editor for the journal ACS Sensors. 